\title{
Targeted drug delivery with modified gamma- Cyclodextrin nanocarriers and MR-guided focused ultrasound triggering
}

\author{
Doudou Xu ${ }^{1 *}$, Lijun Wang ${ }^{1}$, Sandy Cochran², Andreas Melzer ${ }^{1}$ \\ From Current and Future Applications of Focused Ultrasound 2014. 4th International Symposium \\ Washington, D.C, USA. 12-16 October 2014
}

\section{Background/introduction}

NANOPORATION project sets out to explore specific solutions to overcome the current challenges of targeted drug delivery (TDD) to tumours using magnetic resonance imaging guided focused ultrasound (MRgFUS) to cavitate microbubbles (MBs) for increasing cell permeability and to open 'drug nano-capsules' for releasing proven active anticancer drugs directly to the tumour site with reduction of drug dosage needed for the desired therapeutic effect.

\section{Methods}

A novel gamma-Cyclodextrin (gamma-CD) based carrier for encapsulation of doxorubicin (DOX) was synthesized and fully characterized. The encapsulation efficiency was assessed by chemical analysis, in vitro and in vivo. A high-throughput in vitro micro-scale FUS device (sonicator) was designed and applied to cells exposure to carrier-DOX inclusion, in combination with SonoVue ${ }^{\circledR}$ MBs to investigate TDD in monolayer cellular level. Ex vivo and in vivo trials were carried out by clinical ExAblate MRgFUS system (InSightec, Israel) to establish a safe and efficient clinical TDD protocol on small rodents.

\section{Results and conclusions}

The desired gamma-CD based carrier greatly reduced DOX's toxicity in vitro: up to $95 \%$ toxicity reduction in KB human enpidermal carcinoma; up to $92 \%$ toxicity reduction in HCT116 colorectal carcinomar. Cellular DOX uptake was reduced $73 \%$ in muscle, $69 \%$ in kidney, $66 \%$ in liver, $65 \%$ in heart, $62 \%$ in brain, $53 \%$ in lungs as

${ }^{1}$ University of Dundee, Dundee, United Kingdom

Full list of author information is available at the end of the article
$25 \%$ in plasma in vivo. The carrier-DOX inclusion is highly stable under physiological temperature and $\mathrm{pH}$ as well as under a wide range of acidic conditions $(\mathrm{pH}$ 1.0 7.0); the encapsulated DOX is slowly released under hyperthermic conditions (up to $50^{\circ} \mathrm{C}$ ). In the presence of MBs $(0.1 \%, 1 \%, 2.5 \%$ and $5 \%)$ application of FUS with low mechanical indexes $(0.24,0.31$ and 0.53$)$, under which no thermal effect was observed, enhanced up to 3.89-fold of cellular drug uptake for encapsulated DOX in vitro. Optimal setup of MR parameters: $\mathrm{TR} / \mathrm{TE}=$ $3180 / 96.3 \mathrm{msec}$; bandwidth: $10.4 \mathrm{kHz}$; Field of View = $20 \times 20 \mathrm{~cm}$; matrix: $384 \times 384$, NEX: 2 ; slice thickness: $2.0 \mathrm{~mm} / 1.0 \mathrm{sp}$; number of slices: 8 ; frequency direction: SI and the spatial resolution: $0.52 \mathrm{~mm}$; FUS parameters: $4 \mathrm{~W}, 10 \mathrm{sec}$ continued sonication and $45 \mathrm{sec}$ pulsed sonication with $2.5 \mathrm{sec}$ OFF and $0.5 \mathrm{sec} \mathrm{ON}$; temperature increase of $7-10^{\circ} \mathrm{C}$; as well as treatment time frame of $35 \mathrm{~min}$ were identified ex vivo and in vivo, which allowed application of MRgFUS treatments to live mice bearing tumors under anesthesia with full recovery.

Unfortunately, the lack of detectable DOX signal was obtained from the very first pre-clinical trial. However, the study demonstrated the possibility of translation of the constructed gamma-CD derivative to potential clinical use as a delivery vehicle of DOX for combined thermal and mechanical mechanism by clinically applicable MRgFUS, -triggered TDD for cancer therapy.

\section{Acknowledgements (Funding)}

The research leading to these results has received funding from the European Union's Seventh Framework Programme (FP7/2007-2013) under grant agreement $n^{\circ} 230674$ (NANOPORATION) and n²70186 (FUSIMO).

\section{Authors' details \\ 'University of Dundee, Dundee, United Kingdom. ${ }^{2}$ Institute for Medical Science and Technology, Dundee, United Kingdom.}



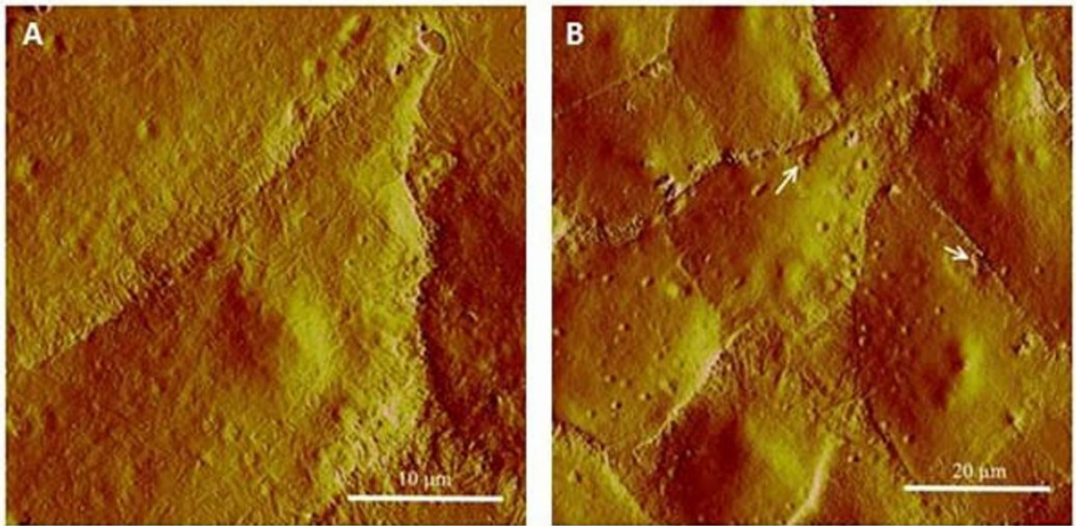

Figure 1 AFM cell surface morphology comparison of KB cells before (A) and after (B) $f=0.4868 \mathrm{MHz}$ sonication with $2.5 \% \mathrm{MBs}$

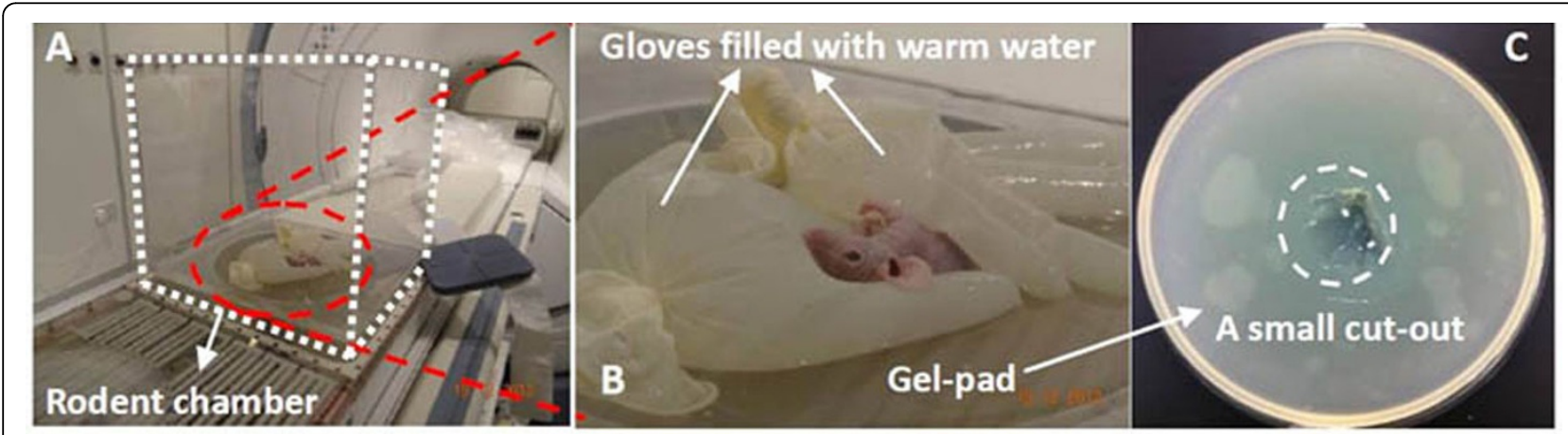

Figure 2 Animal positioning on ExAblate 2000 table inside rodent chamber (A); mouse was protected by gloves filled with water (B); on top of coupling gel-pad with a small cut-out (C)

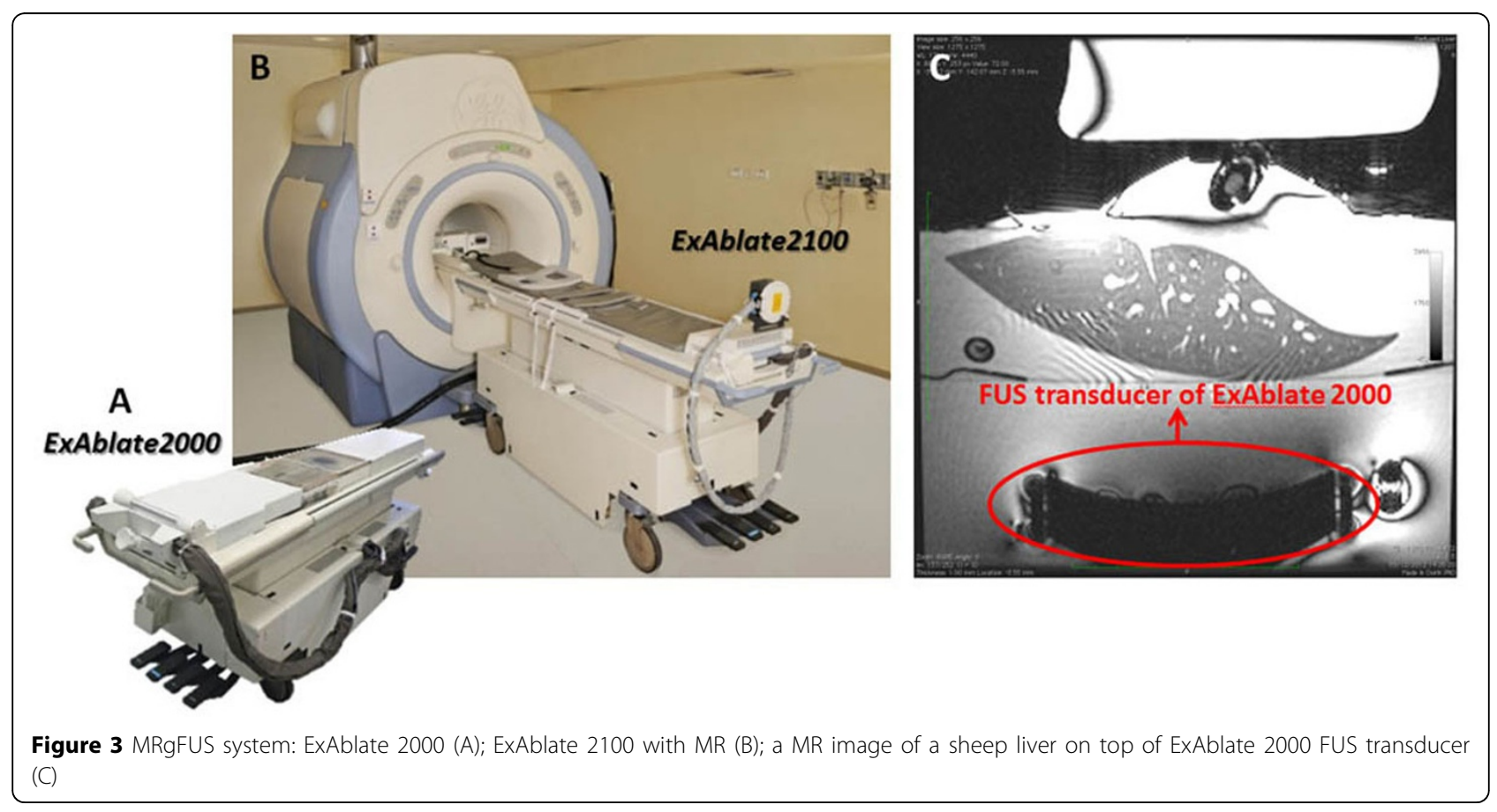




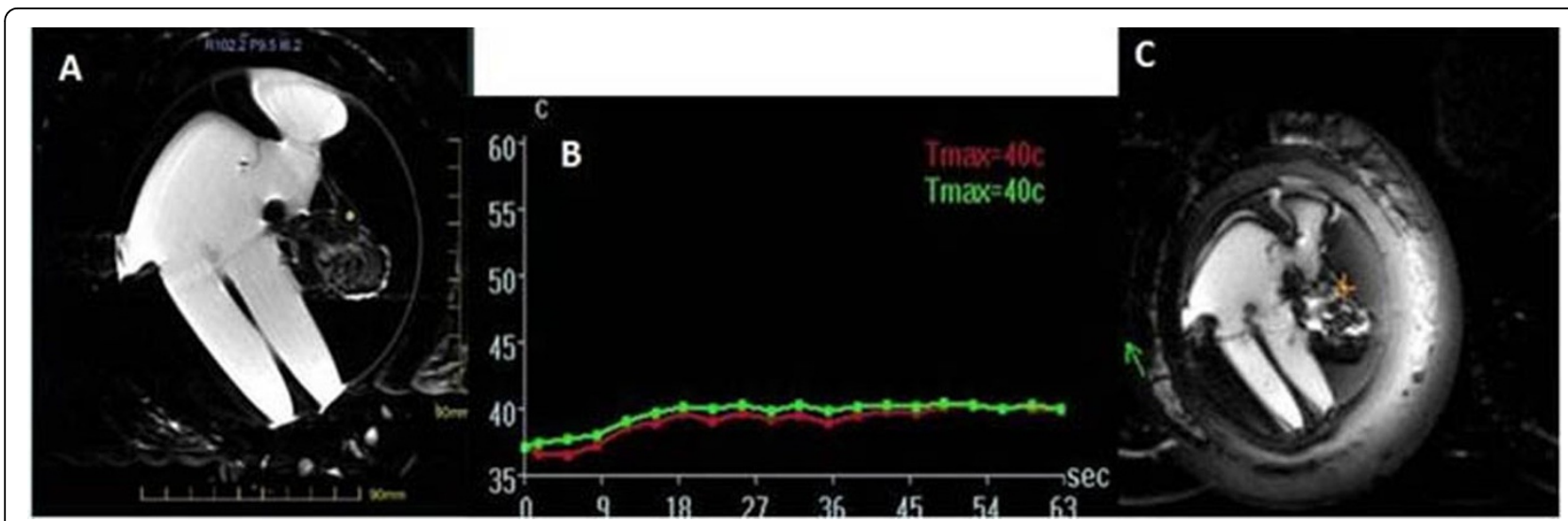

Figure 4 Example of Thermal Monitoring during sonication: Coronal MR image showing planned sonication location (A); temperature graph during sonication (B); the anatomical image acquired during sonication (C)

- Convenient online submission

- Thorough peer review

- No space constraints or color figure charges

- Immediate publication on acceptance

- Inclusion in PubMed, CAS, Scopus and Google Scholar

- Research which is freely available for redistribution 